\title{
National EMS Curricula: Guidelines or Policies for Practice?
}

\author{
Rick Barney, MD, EMT-Paramedic
}

Medical Director, State of Wisconsin USA
As the Medical Director of Emergency Medical Services for the State of Wisconsin, I feel compelled to share the results of our review of the new [U.S.] National Highway Traffic Safety Administration (NHTSA)-sponsored Curriculum for the training of Paramedic-level emergency medical technicians (EMT-P). A Committee of nurse and physician educators from each of the Wisconsin Paramedic Training Centers has been reviewing and evaluating the proposed national paramedic curriculum, line-by-line, since April 1996. As the review process progressed, it became apparent that implementation of the proposed paramedic curriculum would not meet the needs for paramedic education in the State of Wisconsin, and thus, the Committee began a lengthy process of editing and re-writing the material included in the proposed curriculum.

First, after attending several national meetings during the past six months, I was disappointed to find that, other than in Wisconsin, no independent review of the material was being done at a state level. Several states had planned to implement a review, but no significant work has been done. It seems that many people became fatigued with the review task, as the review process necessarily is a long and arduous task: the document is large and contains copious amounts of material. Further, any such review first requires a defined scope of practice. The proposed curriculum never clearly presents the scope of practice that it has been designed to address. And the "National Rollout" is just weeks away. It seems that most of the states have accepted that the National Curriculum Committee knows best, and plan to accept and implement this new curriculum without a comprehensive review of the material in terms of their identified scope of practice for the paramedics in their respective states.

It seems that through this curriculum, the National Highway Safety Administration is attempting to expand the paramedic scope of practice across the entire United States. Our Review Committee has found numerous examples of material that are not consistent with our intended scope of practice for paramedics in Wisconsin. It attempts to require learning of information that is not necessary for the practice of paramedical sciences in the streets. In this context, we find no basis for these changes. Even for our career Paramedics, much of the material is pulled from a wish list that one might use to train physician assistants or nurse practitioners. The street paramedics never will see, identify, or treat many of the relatively rare medical problems that so often are addressed in the curriculum: they are trained to provide truly emergency medical services in the prehospital setting and not to provide many of the services for which material is provided in the proposed Curriculum. Thus, without on-going, continuous use, the material taught certainly will not be retained. Should they really be trained using only physical diagnosis, to identify a dislocation of a shoulder, hip, ankle, or shoulder and attempt to reduce such a possible dislocation or fracture in the field? Is this necessary? Is it dangerous? Would an emergency or acute care physician do such procedures in the prehospital setting? What will be the experience level with such maneuvers in paramedic practices? This will be a waste of time and money.

Moreover, Wisconsin depends on a huge volunteer base to provide emergency medical services. In addition, 
Wisconsin has many rural areas, and it is highly unlikely that the use of much of the knowledge and many of the skills proposed will be used in these settings. As we work to increase meaningful advanced care to rural areas, teaching and maintaining this type of curricular content will be time and cost prohibitive. Have the benfit:cost and cost-effectiveness of much of the new curriculum been considered? And the costs not only will be in terms of economics; they will have a substantial human cost as well. Perhaps, the NHTSA is attempting create a new critter, but this is not our view of the scope of practice for the street paramedic.

The Review Committee also has found some of the information contained in the curriculum to be incomplete or misleading. In addition, there are many objectives that lack corresponding content and some sections in the content do not have corresponding objectives. Much of the content lacks consistency in depth and continuity of detail. Thus, we have begun the large and arduous task of changing much of the information in several of the proposed modules to be more consistent with current and anticipated future practices.

While there are some good portions within the new material, Wisconsin will develop a curriculum that eliminates the "factoid" material, includes all useful information, and truly parallels the standard practice we feel is appropriate for these advanced rescuers. I believe that several States will find it impossible to accept large portions of this new curriculum. With each state doing its own revisions, there will not be a National Curriculum. This has the potential to make testing and reciprocity between States problematic.

Before blindly accepting and implementing the new "National Paramedic Curriculum", I strongly advise every state and country to closely scrutinize the proposed curriculum in terms of the current and anticipated future scope of practice for the advanced rescuers as contained in their overall EMS plan for the future.

[Editor's Note: A detailed critique of the proposed paramedic curriculum for the United States resulting from the comprehensive review will be included in the October issue (Volume 14, Number 4) of Prehospital and Disaster Medicine. M.L.B.] 